Poznańskie Studia Teologiczne 30(2016), s. 141-157.

doi: $10.14746 /$ pst.2016.30.06

Jérémie Fischer ${ }^{1}$

Archives municipales de Villefranche-sur-Saône (adjoint au directeur), France

\title{
Les Juifs vus par un prêtre français en exil: l'antijudaïsme dans les Mémoires de l'abbé Pochard (1796-1830)
}

\section{Introduction}

Prêtre français émigré en Grande-Pologne à partir de 1796, Claude Antoine Pochard (1766-1833)² est le gouverneur des enfants de Józef Skórzewski (1757-1809), staroste de Gniezno, et de son épouse Helena Skórzewska (1766-1846). Grand voyageur de par ses fonctions, le statut social de ses employeurs et le nombre d'élèves à sa charge ${ }^{3}$, l'abbé est aussi un mémorialiste méticuleux. En effet, tout au long d'un exil qui a duré jusqu'à sa mort - soit 41 années dont 37 en Pologne - il a consigné le récit des événements, de ses voyages, de ses rencontres et de ses impressions, avec un remarquable degré de précision.

Les Mémoires ${ }^{4}$ de l'abbé Pochard, non publiés à ce jour, représentent une source nouvelle ${ }^{5}$ pour appréhender la période de la fin du XVIII e et du premier

\footnotetext{
${ }^{1}$ Jérémie Fischer - archiviste professionnel, titulaire d'un Master 2 de recherche en sciences historiques (Université de Strasbourg) et d'un Master 2 professionnel en archivistique (Université Lyon III).

Il travaille à la biographie de l'abbé Pochard et à la transcription intégrale de ses Mémoires, en vue d'une édition scientifique bilingue (franco-polonaise).

${ }^{2}$ Sur la vie et le parcours de l'abbé Pochard, voir J. Fischer, L'adoption d'un prêtre réfractaire comtois par une famille de l'aristocratie polonaise: L'abbé Pochard chez les Skórzewski (1796-1833), in: Les Aristocraties en Europe du Moyen Age à nos jours, red. P. Werly, Strasbourg, 2011, pp. 111-120. Voir également la version polonaise de cet article: J. Fischer, Uchodźca z Francji $w$ wielkopolskiej rodzinie ziemiańskiej. Ksiądz Claude An-toine Pochard u Skórzewskich (1796-1833), „Ecclesia. Studia z Dziejów Wielkopolski” 7 (2012), pp. 175-187. L'auteur termine actuellement la rédaction de la biographie de l'abbé Pochard.

${ }^{3} \mathrm{Au}$ cours de son séjour en Pologne, l'abbé Pochard sera amené à superviser, dans plusieurs villes différentes, l'éducation des cinq fils du couple Skórzewski: Raymond (né en 1791), Hilaire (né en 1792), Ignace (né en 1794), Joseph (né en 1798) et Antoine (né en 1803) ainsi que de leurs cousins Marie Lipska (née en 1804) et Alexis Lipski (né en 1805).

${ }^{4}$ L'auteur de cet article regroupe sous les termes de Mémoires polonais les trois tomes relatifs à la période 1796-1833, tandis qu'il parle de Mémoires suisses pour celui qui est relatif aux années 1792-1796.

${ }^{5}$ Bien que consultables à la Bibliothèque Universitaire de Poznań et aux Archives nationales de Poznań, les Mémoires de l'abbé Pochard n'ont pas encore été publiés à ce jour. L'auteur travaille
} 
tiers du XIX ${ }^{e}$ siècle. Si son témoignage a déjà permis d'aborder le champ de l'histoire sociale, via les thématiques de la noblesse ${ }^{6}$ ou de la mobilité ${ }^{7}$, et d'enrichir, à des échelles différentes, la connaissance de l'histoire d'un village de Grande-Pologne ${ }^{8}$ ainsi que l'histoire politique de la Pologne napoléonienne ${ }^{9}$, il peut également apporter un regard original sur des questions d'ordre religieux.

Dans ce domaine particulier, il s'agira ici d'étudier la vision de l'abbé Pochard sur les Juifs. Si cette thématique est somme toute marginale dans la globalité de ses récits, le mot «Juif » est toutefois mentionné à 55 reprises par l'abbé. Il est donc intéressant d'appréhender le positionnement de ce prêtre français face aux Juifs qu'il rencontre au cours de ses voyages, que ce soit en Allemagne, en Pologne et en Lituanie.

Dans un premier temps, il s'agira de voir que Pochard ne fait que constater leur présence. Il conviendra ensuite de présenter sa stratégie d'évitement de la communauté juive en raison de ses sentiments antijudaïques, pour enfin montrer que malgré sa volonté, certains contacts ne peuvent être évités.

\section{Le constat d'une présence juive dans les villes traversées par l'abbé}

\section{A. Une présence évoquée}

En juin 1796, l'abbé Pochard entreprend le voyage qui doit l'amener en Pologne où il est attendu pour devenir gouverneur dans la famille Skórzewski. C'est en traversant l'Allemagne depuis la Suisse, que la présence de populations juives est pour la première fois évoquée dans ses Mémoires ${ }^{10}$. Ainsi, après être

toutefois dans ce sens en réalisant les transcriptions des quatre tomes qui constituent cette source inédite. Sur les trois tomes conservés par la Bibliothèque Universitaire de Poznań, voir R. Wilgosiewicz-Skutecka, Polska w oczach guwernera Skórzewskich, czyli zapiski księdza Pocharda z lat 1792-1833 w zbiorach Biblioteki Uniwersyteckiej w Poznaniu, „Biblioteka” 2011, nr 15(24), pp. 9-24.

${ }^{6}$ Voir J. Fischer, Les différents visages de la noblesse polonaise dans les Mémoires (1796-1833) de l'abbé Pochard, «Annales du Centre scientifique de l'Académie polonaise des sciences de Paris, volume $17 », 2015$, pp. 257-265.

${ }^{7}$ Voir J. Fischer, De la mobilité contrainte aux contraintes de la mobilité dans les Mémoires de l'abbé Pochard, prêtre français en exil (1792-1833). À paraître dans les actes du colloque international et pluridisciplinaire Dans leurs propres mots, la mobilité dans les écrits personnels et sources orales $X V I^{e}-X X I^{e}$ siècles, organisé par l'Université de Saint Boniface à Winnipeg (Canada), les 27 et 28 août 2015.

${ }^{8}$ Voir J. Fischer, L'abbé Pochard w Kretkowie, czyli zwiqzki księdza emigranta z Francji $z$ tamtejszym dworem i parafia w latach 1810-1833, w: Kretków - właściciele, zabytki, duszpasterze, Poznań 2014, pp. 253-270.

${ }^{9}$ Voir J. Fischer, Le Grand-duché de Varsovie vu par un émigré français ou la relation de cet épisode politique dans les Mémoires de l'abbé Pochard (1806-1815), « Poznańskie Studia Teologiczne $28 »$ (2014), pp. 67-97.

${ }^{10}$ Bien que l'abbé ait vécu et voyagé en Suisse pendant quatre années, il n'est pas surprenant 
passé à Nuremberg, il note que "près de la ville, il y a un village où les Juifs peuvent s'établir ${ }^{11}$ » et qu' « on y trouve à acheter tout ce que l'on veut $»^{12}$. À Leipzig, l'abbé mentionne leur présence parmi d'autres groupes confessionnels:

[...] étant à la promenade, nous avons vu un prêtre grec et le prêtre françois qui est chapelain de l'électeur ${ }^{13}$, nous a dit à ce sujet qu'ils exerçoient leur culte dans cette ville, ainsi que les Juifs et toutes les autres sectes. $[\ldots]^{14}$.

Si à Potsdam l'abbé apprend que « les Juifs [...] sont tolérés ${ }^{15} »^{16}$, lors de son passage à Berlin il formalise le même constat que celui fait plus tôt à Leipzig, mais il précise désormais leur profession commerçante:

[...] C'est dans Berlin que sont les Juifs ${ }^{17}$ qui y ont une synagogue et qui forment la plus grande partie des commerçants ${ }^{18}$. La place près de laquelle ils demeurent est

qu'il n’y ait pas constaté de présence de Juifs. En effet, ces derniers n'ont jamais été très nombreux dans ce pays. Bannis au début du XVII ${ }^{e}$ siècle, ils obtiennent, en 1776, la permission exclusive de s'installer dans deux villages de l'Aarau, Lengnau et Oberendingen. À la fin du XVIII' siècle, au moment où Pochard se trouve en Suisse, seuls 553 Juifs sont recensés dans ces deux villages.

${ }^{11}$ Les Juifs sont interdits de cité à Nuremberg, à l'instar de Brême et d'Augsbourg à la même période. Cf. R. Charpiot, Histoire des Juifs d'Allemagne du Moyen Âge à nos jours, Paris 2009, p. 72 .

${ }^{12}$ Mémoires polonais, t. I, BU UAM, sygn. 115 I/t.1., lettre 3 du 21 juin 1796, p. 57. Les sigles suivants sont utilisés dans les notes de bas de page: $\mathrm{BU}$ UAM $=$ Bibliothèque Universitaire de l'Université Adam Mickiewicz de Poznań ; APP = Archives Nationales de Poznań (Archiwum Państwowe w Poznaniu).

${ }^{13}$ Il s'agit de Frédéric-Auguste III (1750-1827), électeur de Saxe de 1763 à 1806 puis roi de Saxe jusqu'à sa mort.

${ }^{14}$ Mémoires polonais, t. I, lettre 4 du 26 juin 1796, p. 77.

${ }^{15}$ Lorsqu'il arrive au pouvoir en 1740, Frédéric II de Prusse (1712-1786) déclare que toutes les religions devront être tolérées, car chacun de ses sujets a le droit «d'accéder au salut comme il lui plait ». Il prend effectivement des mesures qui vont dans ce sens dans les années suivantes (plus d'entrave à la pratique du culte judaïque, tribunaux identiques pour les Chrétiens et les Juifs etc.). Au-delà de ce principe de tolérance, sa politique consiste à utiliser le sens des affaires de ses sujets juifs aisés, leurs relations et leur pouvoir financier afin de favoriser le développement économique de son royaume. Ainsi, certains se voient confier l'exportation de produits industriels et artisanaux vers la Pologne, certains prennent la tête d'entreprises de filature ou obtiennent le monopole de la production du tabac, tandis que d'autres investissent dans les forges, les fabriques et les manufactures. Cf. R. Charpiot, op. cit., p. 57.

${ }^{16}$ Mémoires polonais, t. I, lettre 5 du 4 juillet 1796, p. 86.

${ }^{17}$ La présence des Juifs à Berlin est attestée depuis 1295. Cf. Le guide culturel des Juifs d'Europe, Seuil 2002, p. 122. En 1786, soit dix ans avant le passage de Pochard dans la ville, cette dernière compte 112000 habitants dont 3372 Juifs répartis en 400 familles. Cf. R. Charpiot, op. cit., p. 59.

${ }^{18}$ De 1730 à 1768 , plusieurs règlements encadrent de manière plus ou moins restrictive la présence des Juifs en Prusse et plus particulièrement leur activité commerciale. Celui de 1730 limite fortement leur activité, tandis que celui de 1750 répartit les Juifs en 6 classes dont les trois premières sont composées de Juifs fortunés et fortement imposés. Il est à noter que ce règlement créée une classe composée de Juifs dit «tolérés » auxquels toute activité commerciale est interdite. Suite 
toute garnie de boutiques. [...] Les Luthériens, les Calvinistes \&c y ont plusieurs temples, les Juifs leur sinagogue dans la Heydereuter $\operatorname{strasse}^{19}[\ldots]^{20}$.

Lorsqu'à Międzyrzecz, Pochard entre sur l'ancien territoire de la Grande-Pologne, dont l'annexion à la Prusse s'est finalisée lors du partage de 1793, il précise que l' «endroit est catholique mais [qu'] il y a aussi des Luthériens et des Juifs $»^{21}$. À Poznań, son constat insiste à nouveau sur la profession marchande des habitants juifs de Pologne:

[...] Les rues sont généralement parlant trop étroites pour la hauteur des maisons, et elles sont presque toujours sales ${ }^{22}$, surtout celles des Juifs qui sont établis dans toutes les villes de Pologne depuis qu'un des rois de Pologne /Kasimirir ${ }^{23} / 24$ a épousé une Juive nommée Esther ${ }^{25}$ et ce sont eux qui commercent le plus dans ce pays ${ }^{26} \cdot[\ldots]^{27}$.

Si l'abbé mentionne dans ses Mémoires les villes où se trouvent des Juifs durant son émigration de 1796, il réitère ce type de précision quatorze ans plus

aux pressions exercées par les guildes et les corporations, le règlement de 1768, restreint l'activité commerciale des Juifs à l'importation de matières premières et à la vente de vêtements. Cf. R. Charpiot, op. cit., pp. 57-58.

${ }^{19}$ Il s'agit de la première synagogue de Berlin, construite en 1714 sur la Heidereuter strasse (aujourd'hui Rosenstrasse). Epargnée par la Nuit de cristal en 1938 car elle avait été réquisitionnée par la poste allemande, elle sera détruite dans les bombardements de 1945. Cf. Le guide culturel des Juifs d'Europe, op. cit., p. 123. Il est à noter qu'en plus de ce lieu de culte, il existe depuis 1778, l'Ecole juive libre de Berlin, fondée par David Friedländer et Isaac Daniel Itzig. Cf. Chistopher Clarck, Histoire de la Prusse 1600-1947, Perrin 2006, p. 266.

${ }^{20}$ Mémoires polonais, t. I, lettre 6 du 11 juillet 1796, pp. 103-104.

${ }^{21}$ Ibid., pp. 108-109.

${ }^{22}$ Dans son ouvrage consacré aux conditions de vie des Juifs en Pologne, Louis Lubliner explique que la malpropreté des habitations des Juifs (et par extension des rues où ils sont installés), s'explique par le fait qu'ils sont contraints d'habiter des quartiers spécifiques où ils souffrent de surpopulation. Cf. L. Lubliner, Des Juifs en Pologne, examen de leur condition sous le point de vue historique, législatif et politique, Bruxelles-Leipzig 1839, p. 120. À ce sujet, voir également la note 51 .

${ }^{23}$ Il s'agit de Casimir III Piast dit le Grand (1309-1370), roi de Pologne de 1333 à sa mort. Souverain tolérant, il accueille les Juifs qui sont pourchassés dans plusieurs pays européens.

${ }^{24}$ Les mots entre // ont été rajoutés dans l'interligne par Pochard.

${ }^{25}$ Le roi Casimir a eu quatre épouses, mais Esther, dont il est question ici, est sa maîtresse. Il l'aurait rencontrée en 1356 et aurait eu trois enfants avec elle. Selon Louis Lubliner, ce sont seulement deux fils (Pelka et Niemiera) qui seraient nés de cette union. Le premier serait mort en bas-âge et le second aurait été assassiné après la mort de sa mère. Cf. L. Lubliner, op. cit., p. 2.

${ }^{26}$ Selon Louis Lubliner, les principales marchandises dont les Juifs font commerce en Pologne à cette époque sont les «boissons spiritueuses », les "bières », les "étoffes de nouveauté », les « friperies », le « papier», les « denrées » et « l'épicerie ». La prédilection des Juifs pour le commerce est l'un des principaux griefs qui leur est fait. Louis Lubliner explique qu'il s'agit avant tout d'une conséquence des nombreuses difficultés faites à ceux qui souhaitent exercer d'autres professions. Cf. L. Lubliner, op. cit., pp. 95-113.

${ }^{27}$ Mémoires polonais, t. I, lettre 6 du 11 juillet 1796, p. 110. 
tard. En effet, en 1810 , son voyage à Vilnius ${ }^{28}$ où il est chargé de placer deux de ses élèves, lui donne l'occasion de constater la présence des Juifs de l'Est de la Pologne, puis de Lituanie ${ }^{29}$. C'est notamment le cas lorsqu'il passe dans la ville de Ciechanowiec:

[...] Ciechanowiec ville noble qui a été incendiée depuis quelques années ${ }^{30}$, dans le milieu de la partie de la ville qui appartient à la Russie ${ }^{31}$ il y a quatre grands bâtimens murés qui servent de magasin et de boutiques aux Juifs, qui y sont en assez grand nombre $[\ldots]^{32}$.

À Białystok, en territoire russe, la présence de marchands et commerçants juifs est également remarquée par Pochard:

[...] il y a des bâtimens murés qui servent de magasin et de boutiques de toutes sortes de marchandises que les Juifs vendent. $[\ldots]^{33}$.

À son retour de Vilnius, au mois de novembre, Pochard prend connaissance d'une rumeur au sujet des Juifs. Celle-ci concerne une pratique frauduleuse à l'encontre des voyageurs étrangers, qui impliquerait le concours de complices juifs:

[...] j'ai couché à Soleczniki ${ }^{34}$ petite ville noble où il y a une poste. L'auberge est assez commode car il y a deux chambres pour les étrangers ; à peine étois-je entré dans la chambre où je devois coucher, après avoir fait racomoder la voiture que la rudesse du chemin avoit endomagée, qu'un seigneur de Lithuanie qui alloit à Vilna ${ }^{35}$, y est entré aussi pour y passer la nuit, peu de tems après est arrivé un visiteur de magazin de fourage qui couroit la poste, et $\mathrm{j}$ 'ai appris de lui que l'on payoit peu de choses, savoir 6 kopeiks $^{36}$ par cheval, ou 12 gros $^{37}$ pour une station mais en revanche souvent on est obligé d'attendre long tems les chevaux de poste parce que, comme

\footnotetext{
${ }^{28}$ Après avoir été sur le territoire de la République des Deux Nations, liant le Royaume de Pologne au Grand-duché de Lituanie de 1569 à 1795, Vilnius est placée sous domination russe et devient la capitale de l'un des gouvernements de l'Empire.

${ }^{29}$ Les Juifs sont présents en Lituanie depuis le milieu du XIV ${ }^{\text {e }}$ siècle. En effet, le duché de Lituanie a accueilli les Juifs expulsés d'Europe occidentale sous prétexte qu'ils étaient responsables de la Peste noire (1347-1352). Cf. S. Champonnois, F. Labriolle, La Lituanie un millénaire d'Histoire, Paris 2007, p. 59.

${ }^{30}$ Un incendie, qui ravage notamment l'hôpital, est attesté le 28 mai 1809.

${ }^{31}$ Si la ville est prussienne de 1795 à 1806, elle fait ensuite partie du duché de Varsovie pour son territoire situé à l'Ouest du Bug, tandis que l'Est est sous contrôle russe.

${ }^{32}$ Mémoires polonais, t. I, lettre 18 du 16 décembre 1810, p. 249.

${ }^{33}$ Ibid., p. 256.

${ }^{34}$ Il s'agit de Šalčininkai, ville située en Lituanie.

${ }^{35} \mathrm{Il}$ est à noter que la forme française de Vilnius utilisée à cette époque (Vilna) se rapproche de la forme allemande (Wilna) ainsi que de la forme polonaise (Wilno).

${ }^{36}$ Le kopeck est la subdivision du rouble qui correspond à $1 / 100^{\text {e }}$ de ce dernier.

${ }^{37}$ Le gros est une monnaie d'argent utilisée pendant le Moyen Age et l'époque moderne et dont le poids et la valeur ont varié selon les régions d'Europe.
} 
l'on paye peu de chose, vu que tous les habitans payent un impôt pour les frais de poste, les maîtres de poste, qui ne sont pas contents du taux fixé pour les chevaux, tâchent d'obtenir davantage surtout des étrangers à ce pays, en disant qu'ils n'ont pas de chevaux, ou qu'ils sont malades ; si s'ennuyant d'attendre, quelque fois des jours entiers, on offre de payer plus cher, ils font conduire par quelques Juifs des chevaux qu'ils disent appartenir à ces Juifs ${ }^{38}$, quoique ce soient ceux de la poste, et on est obligé de convenir d'un prix ordinairement assez considérable avec ces Juifs pour pouvoir continuer sa route $[\ldots]^{39}$.

En 1820, lors d'un voyage à Varsovie l'abbé Pochard traverse Bolimów dont il note qu'il s'agit d'une « petite ville où il y a beaucoup de Juifs » ${ }^{40}$. Si au cours d'excursions ultérieures, l'abbé évoque la présence de Juifs, comme en 1829 à « Bialsk $^{41}[\ldots]$ petite ville assez commerçante, occupée principalement par des Juifs ${ }^{42}$ où il repasse l'année suivante constatant à nouveau qu'il « y a beaucoup de Juifs ${ }^{43}$, il lui arrive aussi, à une reprise, de prendre note de leur absence dans la ville qu'il traverse. C'est ainsi, qu'en 1808, il écrit à propos de Gostyń:

«j'ai passé par la ville de Gostyń où il n'y a aucun Juif chose extraordinaire pour ce pays $^{44} \gg^{45}$.

\section{B. Une présence observée}

Au-delà de la simple évocation, dans ses Mémoires, de la présence de la population juive, l'abbé Pochard est également amené à voir des Juifs et à observer leur mode de vie. C'est à Berlin, sur la fin de son voyage à travers l'Allemagne en 1796, qu'il relate pour la première fois le fait d'avoir des Juifs devant ses yeux:

[...] nous sommes montés en voiture, suivie par une autre où étoient des Juifs. Nous

${ }^{38} \mathrm{Il}$ est à ce propos intéressant de noter que la Diète de 1569 avait formulé à l'encontre des Juifs l'interdiction, sous peine de mort, de pratiquer le commerce de chevaux. Cf. L. Lubliner, op. cit., p. 11.

${ }^{39}$ Mémoires polonais, t. I, lettre 18 du 16 décembre 1810, p. 285.

${ }^{40}$ Mémoires polonais, t. II, APP, zespoł 931, sygn. 2166, voyage de Varsovie 1820, p. 627.

${ }^{41}$ Compte-tenu de l'aire géographique qu'il visite à cette occasion (village de Główczyn en Grande-Pologne), il s'agit vraisemblablement ici de la ville de Błaszki située à proximité, dans la voïvodie de Łódź. D'ailleurs, lorsqu'il y repasse l'année suivante, l'abbé orthographie la ville « Blaszkow » ce qui semble corroborer cette hypothèse. Cf. Mémoires polonais, t. III, BU UAM, sygn. $115 \mathrm{I} /$ t. 3., p. 180.

${ }^{42}$ Ibid., p. 180.

${ }^{43}$ Ibid., p. 200.

${ }^{44}$ Les Juifs représentent un cinquième de la population du duché de Varsovie à cette date. En effet, sur les 2,6 millions d'habitants que compte environ le duché en 1808, 500000 sont des Juifs. Cf. L. Lubliner, op. cit., p. 130.

${ }^{45}$ Mémoires polonais, t. I, lettre 12 du 8 juillet 1808, p. 176. 
étions les deux religieuses ${ }^{46}$ et moi dans une voiture couverte, l'abbé Magnin ${ }^{47}$ est resté avec l'abbé $\mathrm{Clerc}^{48}$. [...] nous avons continué notre route par un chemin très sabloneux et sommes arrivés le mardi matin à Eggersdorf, autre village où j'ai vu les Juifs qui nous suivoient dans la 2 de voiture, faire leur toilette en se lavant bien le visage et la barbe ${ }^{49}$ avant de faire leurs prières en se tournant du côté du levant ${ }^{50}$. Quand ils ont eu fini leurs prières, alors ils nous ont imité et ont déjeuné $[\ldots]^{51}$.

Au début du mois d'octobre 1810, l'abbé observe la présence des Juifs de Varsovie $^{52}$ et constate leur nouvelle implantation dans la ville ${ }^{53}$ :

[...] Je suis parti pour Okienke ${ }^{54}$ où demeure Mr Antoine Byszewski ${ }^{55}$ : pour y aller nous avons passé par la rue royale ${ }^{56}$, qui à présent est la rue des Juifs ${ }^{57}$ qui ont changé d'assez beaux bâtimens en boutiques $[\ldots]^{58}$.

${ }^{46}$ Les religieuses françaises avec lesquelles Pochard voyage à ce moment-là se nomment Delorme et de Gand.

${ }^{47}$ Il s'agit de Jean-François Magnin (1763-1835), prêtre originaire du diocèse de Besançon, comme Pochard, et déporté avec lui en Suisse en 1792. Cf. Base du clergé séculier comtois, XVIII ${ }^{e}-X X^{e}$ siècles (consultable à la Bibliothèque de Grammont et Archives diocésaines de Besançon ; la liste des entrées de la base est consultable en ligne: http://www.introcedo.com/index.php/Base_du_clergé).

${ }^{48} \mathrm{Il}$ s'agit de Jean-Claude Clerc (v. 1751-v. 1806), prêtre également originaire du diocèse de Besançon et déporté en Suisse. Cf. Base du clergé séculier comtois, XVIII ${ }^{e}-X X^{e}$ siècles, op. cit.

${ }^{49}$ Le judaïsme comporte de nombreuses cérémonies de purification rituelle par ablution.

${ }^{50}$ Les Juifs prient en direction de la ville de Jérusalem.

${ }^{51}$ Mémoires polonais, t. I, lettre 6 du 11 juillet 1796, p. 106.

${ }^{52}$ La présence des Juifs à Varsovie est attestée à partir de 1414. En 1792, soit une vingtaine d'années environ avant le passage de l'abbé par la capitale, la communauté juive de la ville représente 6750 personnes soit $10 \%$ de la population totale. Cf. Le guide culturel des Juifs d'Europe, op. cit., p. 367.

${ }^{53}$ Un décret du 16 mars 1809, défend aux Juifs de Varsovie d'habiter dans tous les quartiers de la ville et précise les quartiers et les rues où ils sont autorisés à s'installer. Une exception existe à l'attention des fabricants "importants" mais les artisans restent soumis à cette réglementation. Selon Louis Lubliner, ce sont 20000 Juifs de Varsovie qui ont été contraints de quitter leurs habitations et leurs commerces pour s'installer dans les quartiers désignés. Cf. L. Lubliner, op. cit. pp. 90-91, 120.

${ }^{54}$ Il s'agit d'Okęcie, un quartier de Varsovie situé au sud-ouest de la ville, où se trouve actuellement l'aéroport Frédéric Chopin.

${ }^{55}$ Il s'agit d'Antoni Byszewski (1784-1854), comte de Jastrzębiec. Il est le fils d'Arnold Byszewski (1723-1800) et de Katarzyna Skórzewska (1749-1797), sœur aînée de Józef Skórzewski (1757-1809), père des élèves de l'abbé Pochard.

${ }^{56} \mathrm{Il}$ s'agit de la voie royale (en polonais trakt królewski), célèbre promenade qui permettait aux rois de Pologne, dès le XVI e siècle, de se déplacer du palais royal situé dans la vielle ville, à leur résidence d'été située alors hors des murs de la capitale, selon un axe nord-sud. Aujourd'hui, il s'agit avant tout d'une voie touristique et commerciale.

${ }^{57}$ L'abbé évoque certainement ici le Faubourg de Cracovie (en polonais Krakowski Przedmieście) et la rue du Nouveau Monde (en polonais Nowy Świat) qui composent, entre-autres, la voie royale, ainsi que l'avenue de Jérusalem (en polonais Aleje Jerozolimskie). Le nom de cette dernière provient du quartier juif qui existait à cet endroit au XVIII ${ }^{\mathrm{e}}$ siècle sous le nom de la Nouvelle Jérusalem (en polonais Nowa Jerozolima). Bien que ce quartier juif cesse d'exister en 1776, Pochard emprunte vraisemblablement l'avenue de Jérusalem en 1810, pour se rendre à Okęcie chez Antoni Byszewski.

${ }^{58}$ Mémoires polonais, t. I, lettre 18 du 16 décembre 1810, p. 246. 
La même année, au cours de son voyage en Lituanie, l'abbé est témoin de la présence de la population juive de Hrodna. Il note plus précisément, la particularité de l'apparence des Juives:

[...] Cette ville est assez grande, mais il y a beaucoup de Juifs ${ }^{59}$ ainsi que dans toute la Lithuanie. Ce que j'ai remarqué de particulier par rapport aux Juives, c'est qu'elles sont obligées de se couvrir tout le corps d'un grand linge blanc qui les envellope entièrement ${ }^{60}$, lorsqu'elles sortent de leurs maisons pour aller en ville, je n'ai pas pu en apprendre d'autres raisons, sinon que c'est pour les distinguer des chrétiens. [...] Sa place est grande et garnie d'un de ses côtés des boutiques des Juifs, à l'extrémité desquelles est le corps de garde principal $[\ldots]^{61}$.

\section{L'évitement des contacts}

Si l'abbé est amené à voir des Juifs au cours de ses déplacements, il met tout en œuvre pour ne pas avoir affaire avec eux, que ce soit pour l'hébergement, la restauration ou toute autre chose. À cette fin, il adopte un véritable comportement d'évitement qu'il décrit à plusieurs reprises dans ses Mémoires. La stratégie de l'abbé Pochard consiste d'abord à parcourir des distances plus longues que souhaitées afin de ne coucher que chez des catholiques. Voilà ce qu'il écrit au sujet de son voyage de Varsovie à Nekla en avril 1809:

[...] étant arrivés à Wierzba ${ }^{62}$, parce que nous n'avions pas voulu coucher dans une auberge tenue par un Juif comme le sont presque toutes celles qui sont sur le chemin de Varsovie depuis Cleczewo ${ }^{63}$; nous avons été très mal à notre aise, parce que l'aubergiste qui y étoit alors, n’y étant que depuis la St Adalbert 23 avril, tems auquel dans toute la grande Pologne, les aubergistes et autres locataires changent presque tous les ans, n'étoit pas monté et n'avoit rien pour recevoir les étrangers. Nous avons eu milles peines d'avoir un peu de paille pour nous coucher dessus. Quant à la nourriture, nous nous étions heureusement pourvus de vivres en partant de Varsovie. [...] Nous avons passé outre Kleczew pour le dîner pour ne pas entrer dans une auberge tenue par un Juif quoique le Juif qui nous menoit nous y engageat fort, nous

${ }^{59}$ Hrodna, actuellement en Biélorussie, accueille des Juifs dès le XIV siècle. C'est le grand-duc Witold qui leur accorde le droit de cité en 1389. Au cours des siècles suivants, la ville devient l'un des plus importants foyers de la présence juive dans la région. Au XIX ${ }^{\mathrm{e}}$ siècle la population de Hrodna est composée ŕ 60\% de Juifs. Cf. Le guide culturel des Juifs d'Europe, op. cit., p. 515.

${ }^{60} \mathrm{Au}$ cours des siècles, plusieurs prescriptions vestimentaires ont été imposées aux Juifs de Pologne et de Lituanie en vue de les distinguer des chrétiens. On peut citer celle de porter une casquette jaune (instaurée à la fin du XIV e siècle puis à nouveau au début du XVI $\mathrm{XI}^{\mathrm{e}}$ siècle) ou encore celle qui consiste à avoir un drap rouge de forme ronde sur leurs habits (instaurée au début du XV siècle). Cf. L. Lubliner, op. cit., pp. 3, 6, 10.

${ }^{61}$ Mémoires polonais, t. I, lettre 18 du 16 décembre 1810, pp. 261-262.

${ }^{62}$ Il s'agit du village de Wierzbie.

${ }^{63}$ Il s'agit de la ville de Kleczew. 
sommes arrivés à Soboliec ${ }^{64}$ auberge séparée près d'un bois mais tenue par un catholique $[\ldots]^{65}$.

Les principes stricts de l'abbé l'amènent également à s'informer au mieux sur la route et à prendre avantage des opportunités qui se présentent à lui. C'est ainsi qu'en se rendant à Vilnius, en 1810, l'abbé fait halte à Białystok, où il évite au mieux les auberges tenues par des Juifs:

[...] Nous sommes arrivés de nuit à Białystok et avons eu le bonheur de trouver de la place chez un cuisinier de Mde la princesse de Kracovie ${ }^{66}$, nommé Maliszewski ${ }^{67}$, qu'on nous avoit recommandé à Strabla. Je dis que nous avons eu le bonheur d'y trouver de la place, car il n'y a que lui qui ne soit pas juif chez qui l'on puisse loger. $[\ldots]^{68}$.

Sur le chemin de retour de Vilnius, l'abbé marque une halte durant laquelle il rencontre un curé qui ne lui cache pas son hostilité vis-à-vis des Juifs. En affirmant la nécessité de ne pas avoir de contact avec eux, ce dernier cautionne la stratégie d'évitement de l'abbé:

[...] Kossow ${ }^{69}$ petite ville noble où de trois auberges qu'il y a, je n'en ai pas trouvé une où je pusse mettre mes chevaux, ce qui m'a forcé de recourir au curé de l'endroit pour mettre mes chevaux à l'abri et ma personne. Il m'a reçu très honnêtement mais il m'a paru être peu instruit, il me répétoit à tout moment en polonois ces mots : le Juif chez le Juif, le prêtre chez le prêtre. [... $]^{70}$.

Pour l'abbé, l'un des moyens d'éviter l'hébergement chez un Juif peut également consister à être reçu chez des connaissances ou membres de la famille Skórzewski. C'est ce qui arrive en 1820, alors que Pochard, accompagné de son élève Antoine ${ }^{71}$, est en route pour Varsovie. Le gouverneur et son protégé font une halte à Mroga, chez la comtesse Skarbek ${ }^{72}$, qui les invite finalement

${ }^{64}$ Cette localité n'a pas été identifiée. Peut-être s'agit-il de Słaboludź?

${ }^{65}$ Mémoires polonais, t. I, lettre 16 du 29 avril 1809, p. 223-224.

${ }^{66}$ Il s'agit d'Izabella Branicka (1730-1808), soeur du dernier roi de Pologne Stanislaw Poniatowski (1732-1798) et épouse, à partir de 1748, de Jan Klemens Branicki (1689-1771), l'un des plus grands magnats polonais du XVIII ${ }^{\mathrm{e}}$ siècle. À son sujet, Pochard précise plus loin « la princesse de Krakowie née Branicka, Poniatowska ». Cf. Mémoires polonais, t. I, lettre 18 du 16 décembre 1810 , p. 257.

${ }^{67}$ Il s'agit de Józef Maliszewski, maître cuisinier de la princesse Branicka. Cf. Archiwum Państwowe w Białymstoku, Sumariusz dokumentów kamery w Białymstoku 1796-1807, (2006), pp. 79, 206.

${ }^{68}$ Mémoires polonais, t. I, lettre 18 du 16 décembre 1810, p. 256.

${ }^{69}$ Il s'agit de la ville de Kosów Lacki.

${ }^{70}$ Mémoires polonais, t. I, lettre 18 du 16 décembre 1810, p. 291.

${ }^{71}$ Il s'agit d'Antoni Skórzewski (1803-1855).

${ }^{72}$ Il s'agit de Tekla Urszula Wiktoria Byszewska (1786-1858), cousine d'Antoine. C'est l'une des filles de Katarzyna Skórzewska (1749-1797), sœur aînée Józef Skórzewski (1757-1809), père des élèves de l'abbé Pochard. Son époux est Karol Euzebiusz Skarbek (1760-1818). 
à passer la nuit au château. Ils acceptent volontiers cette proposition qui leur permet de dormir dans une demeure catholique. Lorsqu'il rédige cet épisode dans ses Mémoires, l'abbé ne dissimule pas l'antijudaïsme qui l'anime:

[...] En sortant de la voiture j'avois recommandé au cocher de ne point s'écarter du château afin de pouvoir continuer notre route, si je le jugeois à propos, le bon accueil qu'on m'a fait, a été cause que j'ai oublié entièrement, ce que je lui avois recommandé et ce n'est que long tems après avoir pris le caffé et Mr Antoine le thé, qui nous a été servi par Melle Emilie ${ }^{73}$, qui sait déjà faire avec grâce, les honneurs de la maison, qu'un domestique ayant dit quelque chose à l'oreille de Mde, elle a commencé par me demander si je n'étois venu que pour un instant puisque le cocher n'avoit pas voulu dételer. Je lui ai répondu que non, mais que j'avois oublié de le faire avertir, ce que mon compagnon est allé faire sur le champ, n'étant pas plus fâché que moi de passer la nuit dans le château, au lieu d'aller chez quelque Juif, vu qu'il est difficile de trouver jusqu'à Varsovie d'autres aubergistes, les villes exceptées où d'ordinaire on trouve un hôtel tenu par un chrétien, quoique du tems du Duché de Varsovie ${ }^{74}$, on ait porté un décret pour empêcher les Juifs de tenir des auberges et de distribuer les boissons $^{75}$, ceux-ci, connoissant le proverbe, argent fait tout, n'ont pas négligé dans cette occasion ainsi que dans bien d'autres, d'employer ce moyen pour se soustraire à l'oppression, à laquelle ils ne méritent que trop d'être exposés, à cause de leur fourberie $^{76}$, de leur haine pour les chrétiens qui les porte à employer tous les moyens possibles pour se venger du mépris des chrétiens qu'ils se sont attirés par la mort de Notre Seigneur, par leur entêtement à ne pas avouer que le messie est déjà venu et par leur malpropreté ${ }^{77}$, leur avarice ${ }^{78}$ et d'autres vices qu'on leur reproche avec rai$\operatorname{son}^{79} \cdot[\ldots]^{80}$.

${ }^{73}$ Il s'agit d'Emilia Karolina Józefa Skarbek (1806-1834). Fille de Tekla Urszula Wiktoria Byszewska (1786-1858) et de Karol Euzebiusz Skarbek (1760-1818).

${ }^{74}$ Le duché de Varsovie est l'État créée en 1807 par les Français, suite à leur victoire contre la Prusse, et qui perdure jusqu'en 1814. L'introduction du Code Civil en 1808 a pour conséquence l'égalité des droits et devoirs civils pour tous les habitants sans distinction. En 1814, la constitution du nouveau Royaume du Congrès réaffirme la religion catholique comme religion d'État, toutes les autres n'étant plus que tolérées. Cf. L. Lubliner, op. cit., p. 47.

${ }^{75}$ Le décret dont il est ici question n'a pas pu être identifié. À noter que Louis Lubliner ne mentionne aucune interdiction de ce type dans son ouvrage.

${ }^{76}$ Louis Lubliner note qu'il est reproché aux Juifs d'employer « la ruse et la fourberie dans l'exercice de l'état commercial » et que « ce grief est assez fondé » (p. 96). En revanche, il assure que ce sont les «circonstances qui forcent en quelque sorte les Juifs à user de fourberie » (p. 96). Sans entrer dans le détail, l'auteur explique que ce comportement est « le résultat immédiat de la législation exceptionnelle et vexatoire par laquelle ils sont régis» (p. 105) et que « ruse et fourberie, [sont les] armes ordinaires à tout faible persécuté par le fort» (p. 110). Sur la question de la «fourberie» voir plus en détail: L. Lubliner, op. cit., pp. 96-120.

77 À ce sujet, Louis Lubliner écrit : « chargés des impôts ordinaires et surchargés des impôts extraordinaires, qu'y a-t-il de surprenant à ce que le Juif soit obligé de vivre le plus économiquement possible et même d'une manière parcimonieuse ?». Cf. L. Lubliner, op. cit., p. 120. Voir également la note 21 .

${ }^{78}$ À ce sujet, Louis Lubliner écrit : «Je répète, que le Juif en Pologne fait sagement de vivre avec une grande économie, et d'entasser l'argent dans son coffre-fort, la législation exceptionnelle, 
Si l'antijudaïsme de Pochard n'est pas surprenant compte tenu de son statut d'ecclésiastique catholique ${ }^{81}$, il n'en est pas moins le moteur de sa stratégie d'évitement de la communauté juive sur la route. Mais malgré l'attention de l'abbé Pochard à ne jamais avoir affaire aux Juifs, certains contacts avec eux sont néanmoins inévitables.

\section{Des contacts inévitables}

\section{A. Des contacts choisis par défaut}

Au cours de ses longs voyages, l'abbé Pochard n'est pas toujours en mesure d'éviter tout contact avec les Juifs, notamment lorsqu'il traverse des contrées où cette population est nombreuse. La raison première est qu'il lui faut bien trouver un endroit où faire étape, or il arrive quelque fois que seuls des Juifs tiennent auberge là où il se trouve.

C'est en route pour Varsovie en mai 1804 que l'abbé passe pour la première fois la nuit «dans une auberge tenue par un Juif $»^{82}$. Mais c'est au cours de son voyage à Vilnius à la fin de l'année 1810, que Pochard se trouve le plus souvent en contact avec des membres de cette communauté pour cette raison. À « Podsłupia $^{83}$ », l'abbé et ses deux élèves sont amenés à dormir dans une auberge tenue par un Juif ${ }^{84}$, expérience qu'ils renouvèlent à « Troki ${ }^{85}$ », faute d'avoir pu trouver un «hôtel catholique ${ }^{86}$. C'est là que l'abbé apprend des informations sur les conditions de vie des Juifs de Lituanie:

[...] N'ayant pu y trouver un hôtel catholique nous sommes entrés chez un Juif [...]. À peine étions nous retirés dans notre chambre que le seigneur d'Januszewski ${ }^{87}$ est arrivé à la même auberge. Après avoir été pendant quelque tems sans nous adresser la parole, l'entretien a enfin commencé et sa compagnie nous a été fort agréable. Il nous a appris que $[\ldots]$ dans les auberges, les Juifs n'ont point de taxes pour les

vexatoire, les procédés haineux des gouvernements, lui en font un devoir ». Cf. L. Lubliner, op. cit., pp. 122-123.

${ }^{79}$ Sur les sources de la haine envers les Juifs, voir le chapitre que Louis Lubliner leur consacre dans son ouvrage. Cf. L. Lubliner, op. cit., pp. 51-159.

${ }^{80}$ Mémoires polonais, t. II, voyage de Varsovie 1820, pp. 618-619.

${ }^{81} \mathrm{Il}$ faut notamment rappeler qu'au cours du XVIII ${ }^{\mathrm{e}}$ siècle, plusieurs papes édictent des lois anti-juives. Sur l'antijudaïsme dans l'Eglise catholique voir notamment l'explication faite par Louis Lubiner. Cf. L. Lubliner, op. cit., pp. 51-71.

${ }^{82}$ Mémoires polonais, t. I, lettre 10 du 8 juin 1802, p. 161.

${ }^{83}$ Cette localité, qui se situe entre Hrodna (actuellement en Biélorussie) et Merkinè (actuellement en Lituanie) n'a pas pu être identifiée.

${ }^{84}$ Mémoires polonais, t. I, lettre 18 du 16 décembre 1810, p. 263.

${ }^{85} \mathrm{Il}$ s'agit de la ville de Trakai située en Lituanie.

${ }^{86}$ Mémoires polonais, t. I, lettre 18 du 16 décembre 1810, p. 270.

${ }^{87}$ Cette personne n'a pas pu être identifiée. 
choses qu'ils fournissent $^{88}$; que dans beaucoup d'auberges les seigneurs donnent le foin et la paille à un taux fixé, mais ordinairement assez haut ; que dans toutes les villes et vilages ce sont les Juifs qui font la bierre et le brandwin ${ }^{89}$; que les ouvriers doivent avoir des patentes ${ }^{90}$ pour exercer leur art ou métier ; que les Juifs sont estimés dans ce pays et y ont assez de (b. poids) ${ }^{91}$ /crédit/ [...]. Le lendemain ayant éprouvé ce qu'il nous a dit concernant le prix des choses fournies par les Juifs, vu que celui chez qui nous étions a exigé un prix énorme pour ce que nous avons pris de lui nous nous sommes mis en route $[\ldots]^{92}$.

En novembre 1810, sur le chemin de retour de Lituanie, l'abbé s'arrête chez un Juif. Malgré son ressentiment général contre les Juifs, il reconnaît l'honnêteté de son hôte:

[...] ayant passé par Szczuczyn ${ }^{93}$ où il y [a] un collège de piaristes ${ }^{94}$ j'ai couché à Kamionka ${ }^{95}$ chez le premier aubergiste juif qui se soit trouvé sur mon passage depuis Vilna. Ce Juif a été fort honnête et très raisonable, ce qui est extraordinaire. [... $]^{96}$.

Plus loin sur sa route, l'abbé est à nouveau contraint de passer la nuit chez un Juif. Dans ses Mémoires, il semble que Pochard se sente une fois de plus obligé de se justifier sur le caractère exceptionnel de cette entorse à ses principes:

[...] ayant passé la nuit à Gastkow ${ }^{97}$ dans une auberge murée où étoit un Juif, le seul Juif chez qui j’ai logé depuis Varsovie $[\ldots]^{98}$.

Dix ans plus tard, c'est à nouveau sur la route de Varsovie que l'abbé Pochard est amené à passer la nuit chez un aubergiste juif:

[...] nous sommes arrivés pour la nuit à Podêbice ${ }^{99}$ petite ville où il n'y a que des Juifs qui tiennent auberge. $[\ldots]^{100}$.

${ }^{88}$ Contrairement à ce qui semble se passer sur les territoires lituaniens contrôlés par les Russes, les aubergistes juifs du duché de Varsovie, quant à eux, paient une contribution, le konsens, à laquelle les chrétiens ne sont pas assujettis. Cf. L. Lubliner, op. cit., p. 98.

${ }^{89}$ Le bran de vin est de l'eau de vie.

${ }^{90}$ Une patente est un brevet accordé par une autorité pour établir un droit, un titre ou un privilège.

${ }^{91}$ Les mots suivis de b. ont été barrés par l'abbé Pochard. Ils sont souvent remplacés par un autre mot dans l'interligne.

${ }^{92}$ Mémoires polonais, t. I, lettre 18 du 16 décembre 1810, pp. 270-272.

${ }^{93}$ Il s'agit de la ville de Shchuchyn qui se situe actuellement en Biélorussie.

${ }^{94}$ Ordre de clercs réguliers fondé en 1597 par le prêtre espagnol Joseph Calasanz (1556-1648) et destiné à l'éducation.

${ }^{95}$ Cette localité n'a pas pu être identifiée.

${ }^{96}$ Mémoires polonais, t. I, lettre 18 du 16 décembre 1810, p. 287.

${ }^{97}$ Cette localité, censée se situer entre Łęczyca (voïvodie de Łódź) et Turek (voïvodie de Grande-Pologne), n'a pas pu être identifiée.

${ }^{98}$ Mémoires polonais, t. I, lettre 18 du 16 décembre 1810, p. 293.

${ }^{99}$ Il s'agit de la ville de Poddębice.

${ }^{100}$ Mémoires polonais, t. II, voyage de Varsovie 1820, p. 615. 
Plus loin, au cours du même voyage, l'abbé décide de loger dans une auberge où plusieurs Juifs se sont regroupés:

[...] aussi ai-je eu beaucoup de peines de trouver un gîte pour la nuit et ce n'est qu'à Bronice ${ }^{101}$, éloigné de Varsovie d'un mille, que j'ai trouvé une écurie non occupée par les chevaux de l'armée, mais j'ai trouvé la chambre commune remplie de Juifs rassemblés pour célébrer le sabbat ${ }^{102}$ solennel de l'octave des tentes ou tabernacles et la chambre particulière occupée par un officier polonois en quartier dans cet endroit, ce qui n'a pas peu concouru à m'ôter toute envie d'entrer dans une auberge tenue par un Juif. Espérant que l'officier me donneroit un coin dans sa chambre pour y passer la nuit, j'ai envoyé mon compagnon le prier de m'accorder cette grâce, mais le portrait que l'on en a fait, même son domestique, l'en a empêché, et j'étois d'abord déterminé à passer cette nuit dans la voiture, cependant, après une mûre réflexion, je me suis décidé à m'adresser à lui, malgré sa brutalité, son peu de complaisance, son inurbanité que l'on avoit dépeintes sous des couleurs outrées, et j'ai obtenu sans difficulté, ce que je lui demandois. [...] et enfin il m'a appris, que l'on n'étoit pas trop sûre dans cette auberge, plusieurs vols y ayant été commis depuis peu de jours. Cette dernière nouvelle m'a engagé à recommander aux domestiques d'être sur leur garde. Non content de cet avis, ne pouvant guère dormir, je me suis levé à minuit pour voir si les domestiques faisoient ce que je leur avois recommandé, et j'ai trouvé le laquais dormant profondément dans la voiture, l'ayant réveillé, non sans peine, et ne trouvant pas le cocher, je l'ai fait chercher, il étoit près de la porte de la maison occupé à entendre la musique peu mélodieuse des Juifs et après leur avoir représenté le danger auquel ils s'exposoient en veillant si peu, quoiqu'ils eussent été avertis en particulier, par l'hôte de l'auberge, que c'étoient les soldats qui voloient, je me suis recouché pour peu de tems et m'étant mis en voiture de bonne heure, je suis arrivé à Wo$\operatorname{la}^{103}[\ldots]^{104}$.

Outre la question du logement, c'est celle de l'approvisionnement de l'abbé au cours de ses voyages, qui l'amène à entrer en contact avec des membres de la communauté juive. Ainsi, sur le chemin de Vilnius en 1810, ses provisions étant " épuisées », il est contraint de se rendre chez un aubergiste juif à Brańsk pour $\mathrm{y}$ acheter de quoi manger mais ne trouve finalement rien qui lui convienne ${ }^{105}$. En 1820, sur la route de Varsovie, l'abbé sollicite à nouveau des personnes de confession juive pour son approvisionnement, mais ne voit pas non plus ses besoins comblés:

${ }^{101}$ Il s'agit du village de Bronisze.

${ }^{102}$ Le shabbat est le jour de repos assigné au septième jour de la semaine juive, le samedi, qui commence dès la tombée de la nuit du vendredi soir.

${ }^{103}$ Wola est un village où avait traditionnellement lieu l'élection du roi de Pologne. Il s'agit actuellement d'un quartier de Varsovie.

${ }^{104}$ Mémoires polonais, t. II, voyage de Varsovie 1820, pp. 628-630.

${ }^{105}$ Mémoires polonais, t. I, lettre 18 du 16 décembre 1810, p. 253. 
[...] je ne me suis arrêté qu'à Biniawice ${ }^{106}$ pour rafraîchir les chevaux et faire faire une petite réparation à la voiture. Ici je n'ai pas eu lieu de me réconcilier avec les Juifs qui tiennent auberge, car il n'y avoit que du foin, et j'ai été obligé de m'adresser au meunier pour avoir de l'avoine, ce qui m'a retenu plus que je n'aurois voulu, on ne vouloit ni faire du feu, ni donner de pot pour cuire quelque chose pour notre dîner, et ce n'est qu'après trois heures de halte que j'ai pu me mettre en che$\min [\ldots]^{107}$.

Dans ses déplacements, Pochard fait également appel à des Juifs en raison de leur savoir-faire. C'est notamment le cas en novembre 1810, sur le retour de Vilnius, quand il a recours à un artisan pour réparer sa voiture endommagée:

[... J'ai dîné à Cierzewo ${ }^{108}$ petite ville royale ${ }^{109}$ où j'ai eu bien de la peine de trouver un maréchal pour racommoder une des roues et ce maréchal étoit un Juif. Dans ce pays-ci les Juifs exercent tous les métiers ainsi que dans la Lithuanie. [... $]^{110}$.

De la même manière, en 1821, lors d'un séjour à Bad Reinerz ${ }^{111}$, l'abbé est amené à discuter avec un Juif au sujet du change. Mais finalement, Pochard ne fait pas appel à ses services:

[...] Mde voulant changer l'argent m'a envoyé un Juif qui s'offroit à faire le change, mais dès qu'il m'a eu offert $2 \mathrm{fl}^{112}$ pour l'écu, je suis descendu de la voiture pour prendre des informations de l'agio, n'ayant rien pu apprendre de positif de l'apoticaire à qui je m'étois adressé, vu qu'ordinairement on ne prend guerre intérêt à ce qui regarde des étrangers, j'ai pris le parti de m'en rapporter à ce que me donneroit le maître de l'hôtel de l'Agneau, qui m'ayant offert 3 1/2f par écu, taux que m'avoit désigné notre hôte de Reinerz, je n'ai pas hésité de changer de manière à ne pas me trouver dans la nécessité de changer avec perte. $[\ldots]^{113}$.

La même année, à Dresde, Pochard fait appel à un guide qui s'avère être un Juif francophone, converti au catholicisme:

[...] N'ayant pas trouvé de place chez notre hôtesse Konradi, nous sommes descendus dans une auberge /l'Aigle d'or/, pas des meilleurs, près de l'hôtel de ville, dans une rue étroite, y étant conduit par un Juif devenu catholique qui parloit françois. [...] $]^{114}$.

${ }^{106}$ Il s'agit du village de Bieniewice.

${ }^{107}$ Mémoires polonais, t. II, voyage de Varsovie 1820, p. 627.

${ }^{108}$ Il s'agit de la ville de Czyżew.

${ }^{109}$ En 1738, la ville obtient une franchise de la part du roi Auguste III (1696-1763).

${ }^{110}$ Mémoires polonais, t. I, lettre 18 du 16 décembre 1810, pp. 290-291.

${ }^{111}$ Bad Reinerz est le nom allemand de Duszniki-Zdrój, station balnéaire située en Silésie. À cette date, la ville est sur le territoire du Royaume de Prusse.

${ }^{112}$ Il s'agit de florins, nom donné à différentes monnaies européennes initialement frappées en or puis en argent.

${ }^{113}$ Mémoires polonais, t. II, $2^{d}$ voyage de Dresde, pp. 674-675.

${ }^{114}$ Mémoires polonais, t. II, pp. 708-709. 


\section{B. Des contacts subis}

Comme il a été vu précédemment, la majorité des contacts de l'abbé Pochard avec les Juifs sont de sa propre initiative, étant amené, malgré lui, à prendre une posture de demandeur de service. En revanche, il arrive à de rares occasions que des contacts avec des membres de la communauté juive soient subis par l'abbé. C'est ainsi qu'à la fin de l'année 1810, au cours de son voyage à Vilnius, il se trouve à Hrodna, où il «est pris par des Juifs pour un médecin qu'ils vouloient conduire auprès d'un malade $»^{115}$. Malheureusement, l'abbé ne livre pas plus de détails sur les circonstances de cette situation de malentendu.

Parmi les contacts subis par l'abbé, il arrive que certains soient empreints d'une certaine violence. C'est le cas, toujours à Hrodna au même moment, quand l'abbé se retrouve involontairement face à plusieurs habitants juifs. Dans cette confrontation, qu'il ressent comme une agression de leur part, il répond à son tour par une posture agressive:

[...] À peine la voiture at-elle été arrêtée près de la place, où l'on m'avoit dit que demeuroit un traiteur, que la voiture a été entourée de Juifs dont j'ai eu mille peines de me débarasser et cela non sans les menacer de les frapper de ma canne. Celui que l'on nous avoit indiqué ne tenant plus d'étrangers, nous avons eu bien des maux de trouver le seul traiteur chrétien qui soit dans cette ville, mais enfin nous y avons trouvé de la place pour nous et nos chevaux quoiqu'un peu cher $[\ldots]^{116}$.

Arrivé à Vilnius, l'abbé a de nouveau affaire à des Juifs. Si cette nouvelle confrontation est d'abord subie comme à Hrodna, l'abbé en profite pour engager l'un deux à le guider dans la ville. Mais Pochard finit par se sentir trompé par ce guide, ce qui lui occasionne un fort agacement:

[...] Dès que nous nous sommes arrêtés en ville notre voiture a été entourée, come à Grodno ${ }^{117}$ de Juifs, en ayant pris un pour qu'il me conduisit chez Kriszkiewicz ${ }^{118}$ qu'on m'avoit indiqué à Grodno, comme un bon maître d'hôtel, il m'a conduit chez une juive qui traite. À la première vue j'ai cru que ce n'étoit pas une juive et je me suis laissé conduire par elle dans plusieurs chambres qu'elle m'a montré pour que je choisisse ce qui me conviendroit. M'étant apperçu à son langage qu'elle étoit juive je me suis dépêché de quitter cette maison et peu s'en est falu que je n'ai donné des coups de canne au Juif qui m'avoit conduit chez elle, de dépit de m'être laissé tromper, je suis entré chez un horloger qui demeureoit près de là et lui ai demandé de m'indiquer où demeuroit le traiteur Kriszkiewicz, m'ayant désigné la rue, comme il pleuvoit assez, j'ai prié un jeune homme de me mener dans l'hôtel des quatre nations, il l'a fait d'autant plus volontiers, qu'il devoit passer devant cet hôtel où nous sommes débarqués. [...] $]^{119}$.

\footnotetext{
${ }^{115}$ Mémoires polonais, t. I, lettre 18 du 16 décembre 1810, p. 253.

${ }^{116}$ Ibid., p. 260

${ }^{117}$ Grodno est le nom de Hrodna en russe et en polonais.

${ }^{118}$ Cette personne n'a pas pu être identifiée.

${ }^{119}$ Mémoires polonais, t. I, lettre 18 du 16 décembre 1810, pp. 272-273.
} 


\section{Conclusion}

L'analyse minutieuse des Mémoires de l'abbé Pochard, montre que le prêtre français est mû par un antijudaïsme dont il ne se cache ni dans son comportement ni dans ses écrits. Ce sentiment d'hostilité à l'égard de la religion juive et de ses adeptes n'est pas surprenant vu son statut d'ecclésiastique catholique, son époque et le milieu dans lequel il évolue. Si dès 1796, l'abbé Pochard mentionne la présence des Juifs dans les villes d'Allemagne et de Pologne qu'il traverse pour se rendre chez les Skórzewski, il faut remarquer le fait qu'il ne les stigmatise pas pour autant. En effet, il semble les évoquer moins par animosité que par curiosité ; attitude qu'il ne réserve pas qu'aux Juifs puisqu'il mentionne également les autres groupes confessionnels dont il entend parler sur sa route. De même, lorsqu'il est amené à en observer et à faire part de ses observations par écrit, il le fait sans apporter de jugement personnel.

La dureté des sentiments de l'abbé envers les Juifs est mise au jour en d'autres circonstances, qui sont celles de sa stratégie d'évitement des membres de cette communauté lorsqu'il se trouve dans le cas de voyager en Pologne et en Lituanie à des fins professionnelles principalement. De la répulsion qu'il éprouve à leur encontre résulte une volonté de ne pas avoir affaire à eux dans ce cadre précis, que ce soit pour l'hébergement, la restauration ou tout autre service dont il pourrait avoir besoin sur la route. Cela n'est cependant pas réalisable et l'abbé se voir contraint à plusieurs reprises de dormir chez un Juif ou d'avoir besoin du savoir-faire d'un artisan de confession juive. Si à l'une de ces occasions, il est à noter que l'abbé a l'intelligence de reconnaître l'honnêteté de son hôte juif, il n'en change pas moins son opinion sur les coreligionnaires de ce dernier. Ainsi, l'abbé se sent mal à l'aise en compagnie de Juifs, ce qui n'est pas sans occasionner des quiproquos et des réactions violentes à leur égard.

Les Mémoires de l'abbé Pochard illustrent bien le fait que les professions réservées et le champ économique des Juifs les rendent «incontournables » pour les voyageurs contemporains. Ils vivent donc en interdépendance économique faute de véritables échanges sociétaux.

\section{Jews Seen Through the Eyes of a French Priest in Exile: Anti-Judaism in the Memories of Fr. Pochard (1796-1830)}

\section{Summary}

The article is an attempt to show the leading elements of the attitude towards Jews, the attitude of Father Claude-Antoine Pochard (1766-1833), tutor in the family of a Gniezno governor Joseph Skórzewski. Numerous trips across Polish territories and neighboring countries enabled him to come into direct contact with Jewish issues in Central and Eastern Europe, and his up till now unpublished memoirs shed light on some interesting aspects of the history of European Jews. 
The article consists of three parts. The first part presents direct or indirect contacts of Father Pochard with Jews during his trip to Bavaria, Saxony, Prussia, Wielkopolska, the land annexed by Russia and Lithuania. The second part shows Fr. Pochard's reactions to the presence of Jews dislike and avoiding closer contacts. There is also an attempt to show the difference in the positions of both the Catholic side, represented by Fr. Pochard, and the Jewish side, the climate of hostility and mutual prejudices. The third and last part shows cases where Fr. Pochard revises his views in contact with professionalism and honesty encountered in traveling Jewish innkeepers and merchants, and also other specific moments when there was escalation of tension in his relations with the Jews. The whole article is an interesting panorama of Christian-Jewish relations in the first half of the nineteenth century, when there were economic contacts but no cultural exchange.

\section{Keywords}

Fr. Claude-Antoine Pochard, Jews in XIX century, anti-judaism, anti-Christianity

\section{Żydzi widziani oczyma francuskiego duchownego na wygnaniu: antyjudaizm we wspomnieniach ks. Pocharda (1796-1830)}

\section{Streszczenie}

Artykuł jest próbą ukazania wiodących elementów składających się na postawę wobec Żydów, widoczną u ks. Claude-Antoine Pocharda (1766-1833), guwernera w rodzinie starosty gnieźnieńskiego Józefa Skórzewskiego. Liczne podróże po ziemiach polskich i krajach ościennych pozwoliły mu bezpośrednio zetknąć się z problematyką żydowską w Europie środkowej i wschodniej, a jego dotąd niepublikowane wspomnienia rzucają światło na kilka interesujących aspektów historii europejskich Żydów.

Artykuł składa się z trzech części. W pierwszej z nich dokonano prezentacji przypadków bezpośredniego bądź pośredniego zetknięcia się ks. Pocharda z Żydami w czasie jego podróży po Bawarii, Saksonii, Prusach, Wielkopolsce, zaborze rosyjskim i na Litwie. W drugiej części ukazano reakcje ks. Pocharda na obecność Żydów, sprowadzające się najczęściej do niechęci i unikania bliższych kontaktów. Podjęto również próbę ukazania różnicy stanowisk zarówno strony katolickiej, reprezentowanej przez ks. Pocharda, jak i żydowskiej, klimatu wrogości i wzajemnych uprzedzeń. W trzeciej i ostatniej części ukazano z jednej strony przypadki, w których ks. Pochard rewiduje swoje poglądy w zetknięciu z profesjonalizmem i uczciwością napotkanych w podróży żydowskich oberżystów i kupców, a z drugiej konkretne momenty, w których dochodziło do eskalacji napięć w jego kontaktach z Żydami. Całość artykułu stanowi interesującą panoramę stosunków chrześcijańsko-żydowskich w pierwszej połowie XIX wieku, w której za istniejącą wymianą handlową i kontaktami o podłożu ekonomicznym nie idzie wymiana kulturalna.

\section{Słowa klucze}

ks. Claude-Antoine Pochard, Żydzi w XIX wieku, antyjudaizm, antychrześcijaństwo

\section{Bibliografia}

Base du clergé séculier comtois, XVIII ${ }^{e}-X X^{e}$ siècles (consultable à la Bibliothèque de Grammont et Archives diocésaines de Besançon; la liste des entrées de la base est consultable en ligne: http://www.introcedo.com/index.php/Base_du_clergé). 
Champonnois S., Labriolle F., La Lituanie un millénaire d'Histoire, Paris 2007.

Charpiot R., Histoire des Juifs d'Allemagne du Moyen Âge à nos jours, Paris 2009.

Fischer J., De la mobilité contrainte aux contraintes de la mobilité dans les Mémoires de l'abbé Pochard, prêtre français en exil (1792-1833). À paraitre dans les actes du colloque international et pluridisciplinaire Dans leurs propres mots, la mobilité dans les écrits personnels et sources orales $X V I^{2}-X X I^{e}$ siècles, organisé par l'Université de Saint Boniface à Winnipeg (Canada), les 27 et 28 août 2015.

Fischer J., L'abbé Pochard w Kretkowie, czyli zwiazki księdza emigranta z Francji $z$ tamtejszym dworem i parafia w latach 1810-1833, w: Kretków - właściciele, zabytki, duszpasterze, Poznań 2014, pp. 253-270.

Fischer J., L'adoption d'un prêtre réfractaire comtois par une famille de l'aristocratie polonaise: L'abbé Pochard chez les Skórzewski (1796-1833), in: Les Aristocraties en Europe du Moyen Age à nos jours, red. P. Werly, Presses Universitaires de Strasbourg, Strasbourg 2011, pp. 111-120.

Fischer J., Le Grand-duché de Varsovie vu par un émigré français ou la relation de cet épisode politique dans les Mémoires de l'abbé Pochard (1806-1815), «Poznańskie Studia Teologiczne $28 »$ (2014), pp. 67-97.

Fischer J., Les différents visages de la noblesse polonaise dans les Mémoires (1796-1833) de l'abbé Pochard, «Annales du Centre scientifique de l'Académie polonaise des sciences de Paris, volume $17 », 2015$, pp. 257-265.

Fischer J., Uchodźca z Francji w wielkopolskiej rodzinie ziemiańskiej. Ksiadz Claude Antoine Pochard u Skórzewskich (1796-1833), „Ecclesia. Studia z Dziejów Wielkopolski" 7(2012), pp. 175-187.

Lubliner L., Des Juifs en Pologne, examen de leur condition sous le point de vue historique, législatif et politique, Bruxelles-Leipzig 1839.

Mémoires polonais, t. I, BU UAM, sygn. 115 I/t.1., lettre 3 du 21 juin 1796, p. 57.

Le guide culturel des Juifs d'Europe, Seuil, Fondation Jacques et Jacqueline Lévy-Willard, 2002, p. 122.

Sumariusz dokumentów kamery w Bialymstoku 1796-1807, (2006), pp. 79, 206.

Wilgosiewicz-Skutecka R., Polska w oczach guwernera Skórzewskich, czyli zapiski księdza Pocharda z lat 1792-1833 w zbiorach Biblioteki Uniwersyteckiej w Poznaniu, „Biblioteka” 2011, nr 15(24), pp. 9-24. 